\title{
The Same Formation Mechanism of Surface Pits for both a- and c-plane GaN
}

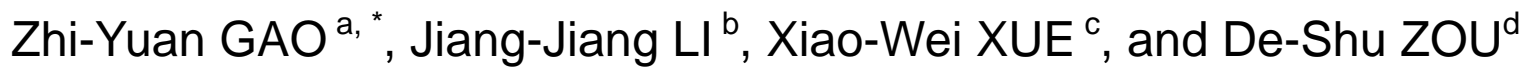

Key lab of Photoelectronic Technology, Beijing University of Technology, Beijing, China, 100124

azygao@bjut.edu.cn, blijianjiang@emails.bjut.edu.cn, 'xwxue@emails.bjut.edu.cn, d449351392@qq.com

*Correspondingauthor 13488717739

\begin{abstract}
Keywords: Screw dislocation, Surface pit, Frank theory,a-plane GaN,c-plane GaN,Island coalescence
\end{abstract}

\begin{abstract}
Surface pits in a-plane GaN have long been considered as a result of island coalescence in literature, while pits in c-plane GaN are the surface termination of screw dislocations. It is found in the paper that small surface pits on a-plane GaN also terminate perfect screw dislocations and partial dislocations (PD), which is due to the intrinsic nature of the spiral growth of screw dislocations. This resulthas demonstrated that a-plane $\mathrm{GaN}$ also follows the prediction based on Frank's classical theory of dislocated crystals. Moreover, although caused by dislocation, the relative size of the small pits will be affected by the kinetic process of the atomic reactions on the surface through V/III ratio variation. Meanwhile, large hexagonal surface pits are observed on c-plane GaN grown under low V/III ratio, which is formed under the same mechanism as the large triangular or pentagonal pits on a-plane GaN surface. During the island coalescing, the lateral growth of the inclined facets are in competition with the vertical growth in the epitaxial orientation, so incomplete island coalescence will produce large surface pits for both a- and c-plane GaN. Under this mechanism, a triangular pit of a-plane GaN could be transformed into a pentagonal pit upon increasing temperature.
\end{abstract}

\section{Introduction}

Since the ground-breaking discovery of the two-step growth procedure for c-plane(0001)GaN in the early 90s[1], there has been extensive research and development in the field of GaN heteroepitaxy, which paved the way for the successful commercialization of efficient blue light-emitting diodes(LEDs)[2]. To improve the device's optical performance further, no polar GaN have attracted the attention of researchers because they could reduce the polarization-induced field [3]. However, when the growth orientation deviated from the c-axis [0001], structural defects increased[4], and attaining smooth surface became difficult. Large surface pit is one of themorphological imperfections that frequently encountered in a-plane(11 $\overline{2} 0)$ GaN growth[5-7].It refers to the inverted triangular pyramid pits that bound by one vertical $(000 \overline{1})$ and two inclined $\{10 \overline{1} 1\}$ facets[8]or,in some studies, pentagonal shapes composed of $\{10 \overline{1} \overline{2}\},\{20 \overline{2} 1\}$, and $\{11 \overline{2} 2\}$ facets[9, 10]. Reducing the V/III ratio could eliminate these defects; therefore, most researchers considered them as a result of incomplete island coalescence [5, 6,8-12], which was due to the increased vertical growth of the $(11 \overline{2} 0)$ facet and decreased lateral growth rate of the inclined facets, such as $\{10 \overline{1} 1\}$ and $(000 \overline{1})[6,8]$.

In fact, pits have been commonly observed on the surfaces of a wide variety of materials, including metals, high $\mathrm{T}_{\mathrm{c}}$ superconductors and $\mathrm{SiC}[13]$. Pits have also been observed on the c-plane GaN surface with an inverted hexagonal shape[14-25]. Tremendous research has been focused on the surface pits in In GaN/GaN MQW structures[19-22], AlGaN/GaN hetero structures[23,24], and GaN grown at low temperature[15, 16,18].However, the formation mechanism of these pits seemed to be a different process compared with that of a-plane GaN. In general, researchers accepted that the pits formed at the surface termination of screw-component threading dislocations (TD)[15-18, 20, 22, 25], as evidenced by cross-sectional TEM. The pits were related to the spiral growth 
surrounding the dislocation core[15, 16, 18, 26], which was theoretically described by Frank ${ }^{[26]}$, and extended with the surface kinetic model[19, 24, 27]in the case of doping atoms.

It deserves further consideration as to why the same material simply having different epitaxial growth directions has such a difference in the formation mechanism of surface pits. If a screw dislocation results ina pit on the surface of c-plane GaN, why it does not behave the same on an a-plane surface? Spiral growth is an intrinsic property for screw dislocation whose Burgers vector is parallel to its dislocation line. On the other hand, both surfaces are fabricated through atwo-step growth procedure; therefore, why does the island coalescence process promote surface pits for a-plane GaN but not for c-plane GaN? The anisotropic crystallographic difference is one possible explanation because c-plane $\mathrm{GaN}$ at least has crystal symmetry within the basal plane whereas a-plane GaN has in-plane anisotropy. However, incomplete island coalescence is attributed to the unadjusted ratio of growth velocities along the in-plane direction vs. the vertical direction; therefore, the in-plane anisotropy of a-plane GaN could only contribute to the asymmetric shape of the pit and the stripe feature but will not determine whether there is a pit or not.

In this study, through a careful examination of the SEM images of a-plane GaN samples, we found that there were some small pits hidden in the background, which might be ignored because they may be taken as a miniature of the large pit. However, in the cross-section TEM analysis, the small pits were found to be associated with screw dislocation, which was the same as that in the case of the c-plane GaN film. Meanwhile, large hexagonal surface pits were found on c-plane GaN grown under a low V/III ratio, which showed similar features in the cross-sectional TEM images as the large triangular pits in a-plane GaN. Therefore, a systematic study of the growth mechanisms of surface pits on a-plane and c-plane GaN film is underway.

\section{Experiment}

The undoped GaN samples were grown by metal organic chemical vapor deposition (MOCVD) using a two-step growth procedure with one low-temperature GaN buffer layer. Triethylgallium (TEG) and $\mathrm{NH}_{3}$ were used as the Ga source and $\mathrm{N}$ source, respectively, and $\mathrm{H}_{2}$ was used as a carrier gas. The reactor pressure was 40 Torr, and the buffer layer temperature was $450^{\circ} \mathrm{C}$. The a-plane and c-plane GaN samples were grown together in the same chamber on different substrates, i.e., c-plane sapphire substrate for c-plane $\mathrm{GaN}$, and r-plane sapphire substrate for a-plane GaN. The temperature of the high-temperature (HT) GaN layer was varied from $865^{\circ} \mathrm{C}$ to $950{ }^{\circ} \mathrm{C}$, and the V/III ratio was varied from 1940 to 2945 . Each time, only one parameter was changed while the others were kept unchanged. The thickness of the HT GaN was approximately $1 \mu \mathrm{m}$.

A high-resolution field-emission scanning electron microscope (FESEM) was used to obtain a clear surface morphology, and an atomic force microscope (AFM) was used for assistance. Cross-sectional transmission electron microscopy (TEM) analysis was used to study the structure origins of the surface pits. The TEM specimens were prepared by means of a focused ion beam (FIB).The defect type was visually distinguished by taking images under different diffraction vectors $\vec{g}$ in the same region according to the invisible criteria[28]. Perfect dislocations were invisible when $\vec{g} \cdot \vec{b}=0$, where $\vec{b}$ is the Burgers vector. PDs disappeared when $\vec{g} \cdot \vec{b}<\frac{1}{3}$, and stacking faults (SFs)were also invisible when $\vec{g} \cdot \vec{R}=$ oor an integer, where $\vec{R}$ is the displacement vector. Specifically, when $\vec{g}=11 \overline{2} 0$, the visible defects were perfect dislocations with $\vec{b}=$ $\pm \frac{1}{3}[11 \overline{2} 0]$ (for the a-plane sample, it was screw type, whereas for the c-plane sample, it was edge type), mixed type dislocations $\left(\vec{b}= \pm \frac{1}{3}<11 \overline{2} 3>, \vec{b}= \pm \frac{1}{3}[\overline{1} 2 \overline{1} 0]\right.$ and $\left.\vec{b}= \pm \frac{1}{3}[\overline{2} 110]\right)$, and PDs (Frank-Shockley type with $\vec{b}= \pm \frac{1}{6}[20 \overline{2} 3]$ and $\vec{b}= \pm \frac{1}{6}[02 \overline{2} 3]$, Shockley type with $\vec{b}=$ $\pm \frac{1}{3}[10 \overline{1} 0]$ and $\left.\vec{b}= \pm \frac{1}{3}[01 \overline{1} 0]\right)$. When $\vec{g}=0002$, the visible defects were perfect dislocations with $\vec{b}= \pm[0001]$ (for the a-plane sample, it was edge type, whereas for the c-plane sample, it was screw type), mixed type dislocations $\left(\vec{b}= \pm \frac{1}{3}<11 \overline{2} 3>\right.$ ) and PDs (Frank-Shockley type with 
$\vec{b}= \pm \frac{1}{6}<20 \overline{2} 3>$ and Frank type with $\left.\vec{b}= \pm \frac{1}{2}[0001]\right)$. When $\vec{g}=1 \overline{1} 00$, the visible defects were basal plane stacking faults (BSFs, $\mathrm{I}_{1}$ type with $\vec{R}= \pm \frac{1}{6}<20 \overline{2} 3>$ and $\mathrm{I}_{2}$ type with $\vec{R}= \pm \frac{1}{3}<$ $1 \overline{1} 00>$ ), PDs (Frank-Shockley type with $\vec{b}= \pm \frac{1}{6}<20 \overline{2} 3>$ andShockley type with $\vec{b}= \pm \frac{1}{3}<$ $1 \overline{1} 00>$ ), and mixed dislocations ( $\vec{b}= \pm \frac{1}{3}[\overline{1} 2 \overline{1} 3], \vec{b}= \pm \frac{1}{3}[\overline{2} 113], \vec{b}= \pm \frac{1}{3}[\overline{1} 2 \overline{1} 0]$ and $\vec{b}= \pm \frac{1}{3}[\overline{2} 110]$ ). In addition, prismatic stacking faults (PSF, $\vec{R}= \pm \frac{1}{2}<101 \overline{1}>$ ) were observed as an inclined straight line near the[0001] zone axis, whereas PSFs were a contrast band near the [1]̄00] zone axis.

\section{Experimental Results}

For a-plane GaN, large triangular surface pits were easily seen scattering in Figure 1(a), bounded by the inclined $\{10 \overline{1} 1\}$ and vertical $(000 \overline{1})$ facets, with edge lengths in the range of $500 \mathrm{~nm} \sim 2 \mu \mathrm{m}$ in the $[1 \overline{1} 00]$ direction and a density on the order of $10^{6} \mathrm{~cm}^{-2}$.It was interesting to find that under high temperature, the pits became pentagonal-shaped, bounded by the $\{10 \overline{1} \overline{2}\},\{11 \overline{2} 2\}$, and $\{20 \overline{2} 1\}$ facets, as shown in Figure1(b).As summarized in Table 1, the density for both the triangular and pentagonal pits reduced under low V/III ratio, indicating that theV/III ratio controlled the surface pit density, whereas the temperature controlled their shape. If we looked carefully into the background, there were some small triangular surface pits hidden with an average size of $50 \mathrm{~nm}$ and a density on the order of $10^{8} \mathrm{~cm}^{-2}$.Figure 1(c) shows the enlarged view of a small triangular pit. In addition, stripe features along the c-direction could be observed, which apparently resulted from the disparity of the growth rates along the c- and m-directions $[1 \overline{1} 00][5,6]$ within the growth plane. Moreover, there are many tiny dark spots decorating the strips and spreading in the whole image, which had a density on the same order as the PDs $\left(10^{10} \mathrm{~cm}^{-2}\right)$. The density of these tiny spots together with the pits of tens of nanometers in diameter increased as V/III ratio increased, which had the same trend as the large surface pits, as plotted in Figure 2(a) and 2(b).

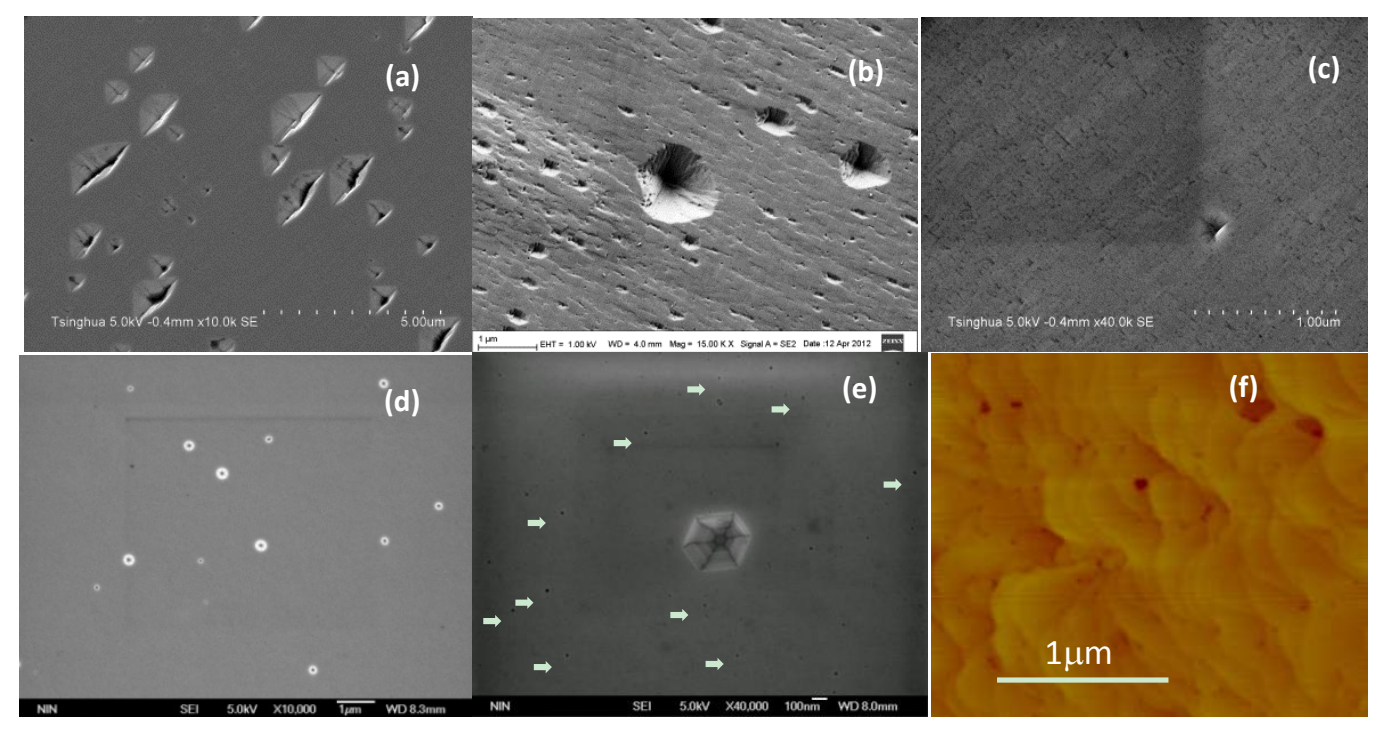

Fig.1.SEM images of the surface morphologies of a-plane GaN and c-plane GaN. (a) Triangular pits scatters on a-plane GaN surface, (b) pentagonal pits observed on the surface of high temperature a-plane GaN sample. (c) Enlarged view of a single small triangular pit of 100nm in diameter, with surrounding many small dark spots of pits of diameter less than 5nm. (d) Hexagonal surface pits on c-plane GaN grown under low V/III ratio. (e) Enlarge view of a single large hexagonal pit, with small hexagonal pits surrounding indicated by the white arrows, for comparison, (f) is a $2 * 2 \mu \mathrm{m}$ AFM image of the typical surface morphology of c-plane GaN, showing small surface pit at the termination of surface steps. 
For c-plane GaN, large hexagonal-shaped surface pits were observed on the samples grown under low V/III ratio, as shown in Figure 1(d), with a density in the range of $10^{5} \mathrm{~cm}^{-2}$, an average diameter of $200 \mathrm{~nm}$, and the largest diameter approaching $1 \mu \mathrm{m}$. Figure 1(e) shows the enlarged view of a single large pit, which was bounded by the $\{10 \overline{1} 1\}$ facets. There were also many small pits in the background with a density of $10^{8} \mathrm{~cm}^{-2}$ and size varied from several nanometers to tens of nanometers, which should correspond to the dark spots that are typically observed in an AFM image. For comparison, Figure 1(f) shows an AFM image of the small pits at the intersections of the atomic surface steps. As summarized in Table 1 and plotted in Figure 2(c) and 2(d), increasing the V/III ratio, the large pits vanished first, resulting in a smooth surface in the SEM image. However, when observed under AFM, the step-pitted morphology was still found but with reduced density.

Table 1 Variation of surface pit density and size under different growth condition of a- and c-plane $\mathrm{GaN}$

No. V/III T $\left({ }^{\circ} \mathrm{C}\right) \quad$ pit density of a-plane $\left(\mathrm{cm}^{-2}\right)$ pit density of c-plane $\left(\mathrm{cm}^{-2}\right)$ $\mathrm{d} \geq 500 \mathrm{~nm} \quad \mathrm{~d} \leq 50 \mathrm{~nm}$ shaped $\geq 200 \mathrm{~nm} \quad \mathrm{~d} \leq 20 \mathrm{~nm}$ shape

$\begin{array}{ccccccc}\text { A } & 2945 & 965 & 9.0 * 10^{6} & 3 * 10^{10} \text { Pentagonnot found } 3.50^{*} 10^{8} & \text { Hexagonal } \\ \text { B } & 2945915 & 9.1 * 10^{6} 2 * 10^{10} \text { Triangle not found } 1.25^{*} 10^{8} \text { Hexagonal } & \\ \text { C } & 2945 & 865 & 9.7^{*} 10^{6} & 1.5^{*} 10^{10} \text { Trianglenot found } 1.50 * 10^{9} & \text { Hexagonal } \\ \text { D } & 2342 & 965 & 3.5^{*} 10^{6} & 2 * 10^{10} \text { Pentagonnot found } 1.00 * 10^{9} & \text { Hexagonal } \\ \text { E } & 2342 & 915 & 3.5^{*} 10^{6} 1 * 10^{10} \text { Triangle not found } 1.50^{*} 10^{8} \text { Hexagonal } & \\ \text { F } & 2342 & 865 & 1.0^{*} 10^{6} & 1 * 10^{10} \text { Pentagon not found } 2.50 * 10^{9} & \text { Hexagonal } \\ \text { G } & 1940 & 915 & 9.0 * 10^{5} & 5 * 10^{9} \text { Triangle } & 1 * 10^{5} & 1.50 * 10^{9} \text { Hexagonal }\end{array}$
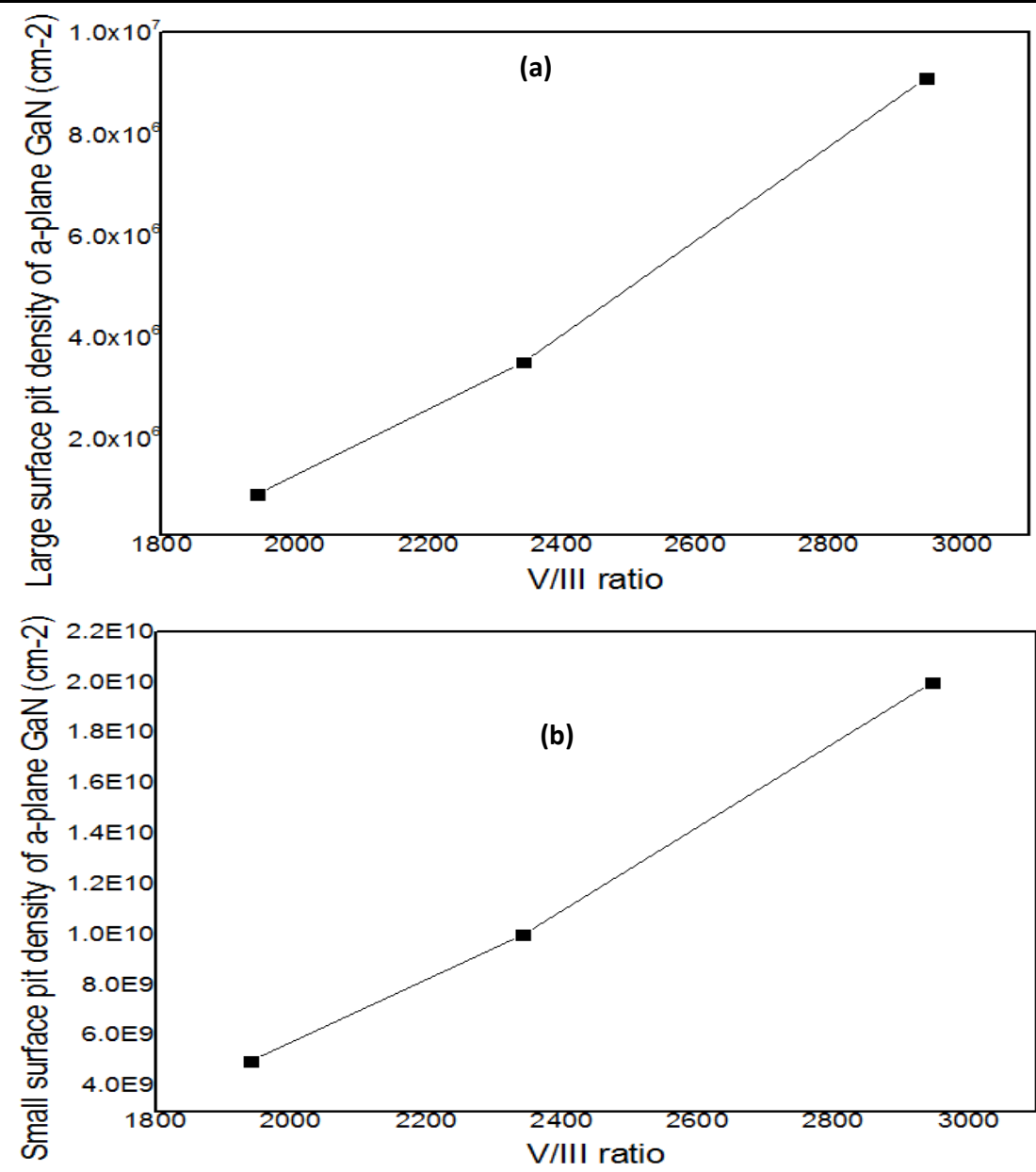

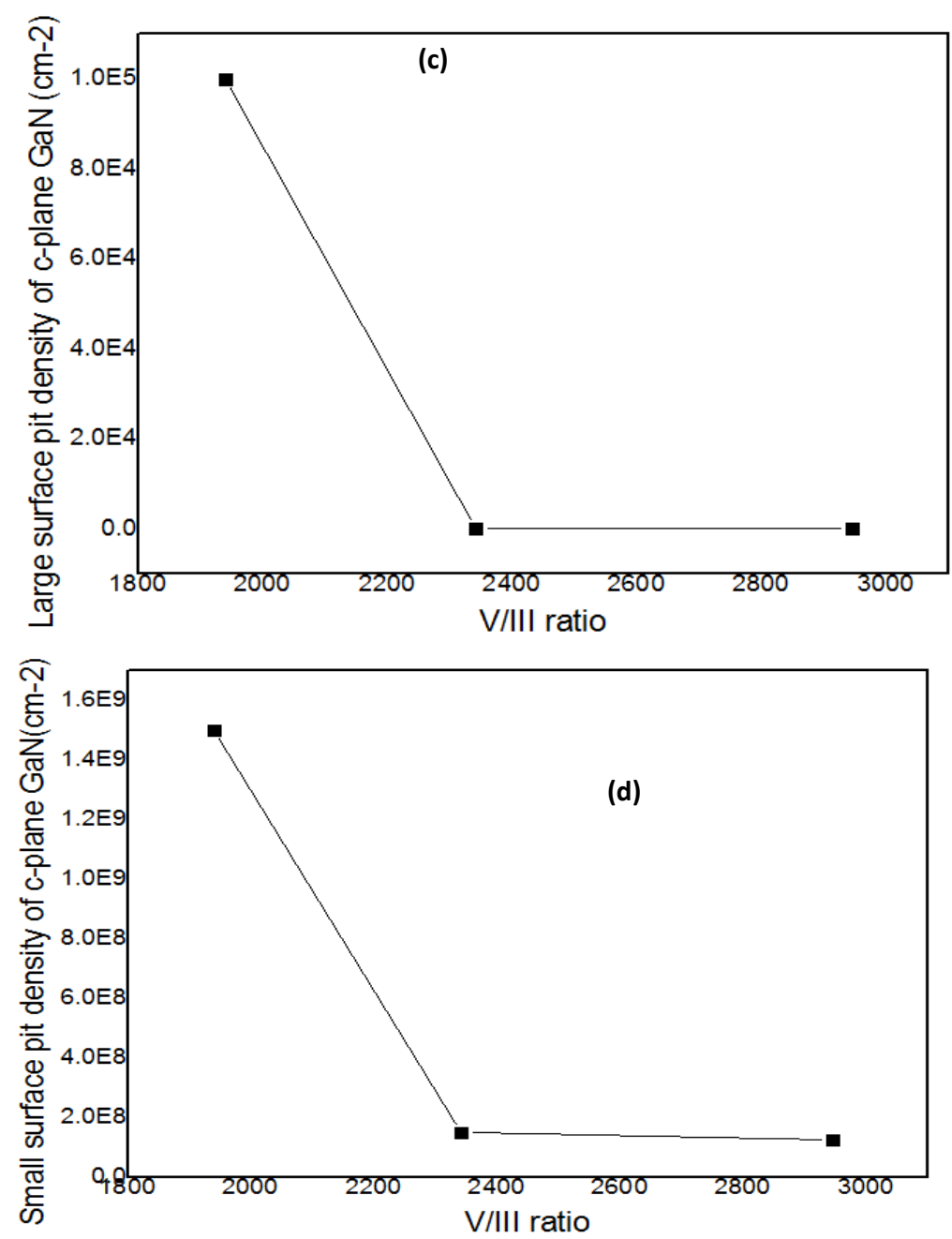

Fig.2. Variation trends of (a) large surface pit density and (b) small surface pit density with V/III ratio under $915^{\circ} \mathrm{C}$ for a-plane $\mathrm{GaN}$, and (c) large surface pit density and (d) small surface pit density with V/III ratio under $915^{\circ} \mathrm{C}$ for c-plane GaN, plotted with the data in Table 1.

The small surface pits for both a-and c-plane GaN were associated with screw component dislocations, as observed in the TEM image in Figure 3. For a-plane GaN, cross-sectional weak beam dark-field images were obtained in the same pit region but using (a) $\vec{g}=11 \overline{2} 0$ and (b) $\vec{g}=0002$ near the $[1 \overline{1} 00]$ zone axis. A defect line could be observed that was associated with the small surface pit in $\vec{g}=11 \overline{2} 0$, whereas it was out of contrast in $\vec{g}=0002$. According to the invisible criteria, perfect screw dislocations can only be observed under $\vec{g}=11 \overline{2} 0$, perfect edge dislocations can only be observed under $\vec{g}=0002$, and mixed dislocations and PDs should appear in both of the two diffraction conditions. The dislocation line that only appeared in $\vec{g}=11 \overline{2} 0$ was identified to be pure screw dislocation. To further determine whether this sized pit was also associated with BSF, which is the commonly observed defect in a-plane GaN, another sample was prepared to obtain images using (c) $\vec{g}=11 \overline{2} 0$ and (d) $\vec{g}=1 \overline{1} 00$ in the same region near the [0001] zone axis because BSF can only be observed under $\vec{g}=1 \overline{1} 00$. However, no defect contrast was observed in the image of $\vec{g}=1 \overline{1} 00$, whereas a screw dislocation line could still be observed in $\vec{g}=11 \overline{2} 0$. Therefore, perfect screw dislocation was the only source for the pits with diameter of tens of nanometers. The pit with extreme small size in the SEM image in figure 1(c) is difficult to identify in the cross-sectional TEM image. In the plan-view image, TEM sample needs to be made very thin to get a clear image contrast of the pit. Inset Figure 3(d) is the plan view TEM image with $\vec{g}=0002$ of the smallest triangular pits, as indicated by the black arrows. The pits were located at the end-on positions of the PDs, demonstrating their connection. 
Figures 3(e) and 3(f) are the cross-sectional weak beam dark-field TEM images for c-plane GaN, obtained in the same pit region but using (e) $\vec{g}=0002$ and (f) $\vec{g}=11 \overline{2} 0$ near the [1 $\overline{1} 00$ ] zone axis. A sharp dislocation line could be observed that was associated with the small surface pit in $\vec{g}=0002$, but became indistinct in $\vec{g}=11 \overline{2} 0$. The bright blurred area under the pit in Figure 3(f) is not the defect contrast because the contrast is not sharp and not a regular shape. According to the invisible criteria, screw dislocations of c-plane GaN can only be observed under $\vec{g}=0002$, edge dislocations can only be observed under $\vec{g}=11 \overline{2} 0$, and mixed dislocations should appear in both of the two diffraction conditions. Because the dislocation line only appeared in $\vec{g}=0002$, it was identified to be perfect screw dislocation. It may be noticed that there were many other screw dislocation lines that were not connected with pits in the image, which was because their connected pits were not in the small cross-sectional sampling area, or in another word, those pits have luckily not been cutting through.
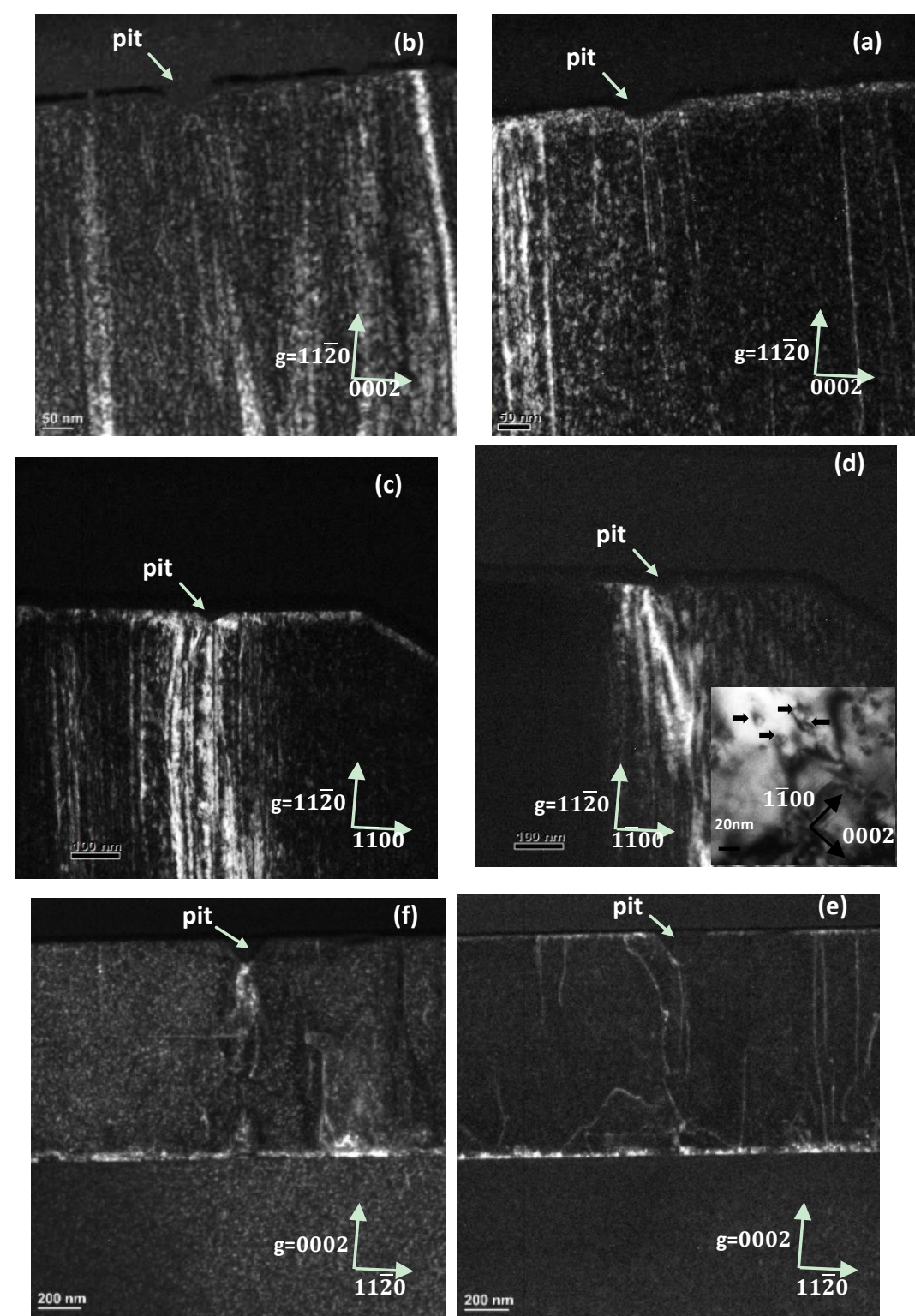

Fig.3. Cross-sectional weak beam dark-field TEM images of small surface pits for a-plane GaN (a) (d) and c-plane GaN (e) (f). Images were taken in the same region of one small surface pit in a-plane GaN using (a) $\vec{g}=11 \overline{2} 0$ and (b) $\vec{g}=0002$ near the [1 $\overline{1} 00]$ zone axis to determine perfect screw dislocation connection, and another pit using (c) $\vec{g}=11 \overline{2} 0$ and (d) $\vec{g}=1 \overline{1} 00$ near the [0001] zone axis to exclude BSF connection.Inset is the plan-view two-beam bright-field TEM image of a-plane GaN under $\vec{g}=0002$ near the [11 $\overline{2} 0]$ zone axis. The small surface pits 
are indicated by the black arrows. Cross-sectional weak beam dark-field TEM images of small surface pits for c-plane GaN using (e) $\vec{g}=0002$ and (f) $\vec{g}=11 \overline{2} 0$ near the [1 $\overline{1} 00]$ zone axis to determine perfect screw dislocation connection.

The large surface pit for both a-plane and c-plane GaN seemed to be built by two blocks of grains with twist and/or tilt in between them, which was evidence of nucleation island coalescence. Figures 4(a) and 4(b) show the cross-sectional weak beam dark-field TEM images of a-plane GaN obtained in the same region of a large surface pit but using (a) $\vec{g}=11 \overline{2} 0$ and (b) $\vec{g}=10 \overline{1} 1$ near the [011̄1] zone axis, which revealed all types of defects including partial and mixed dislocations, as well as BSF. Beneath the pit, there was a strong image contrast at two sides of the $\{20 \overline{2} 1\}$ facet, which was produced by differing diffraction conditions in the two neighboring grains, indicating that the two grains had a twist and/or tilt in between them. The dislocations bent strongly at the interface of the two grains. The images revealed that when two adjacent nucleation islands met, the $\{20 \overline{2} 1\}$ facet of one island met the $\{10 \overline{1} \overline{2}\}$ facet of the other island. Because both of the facets were slow growing planes, they maintained their growth fronts in the lateral direction and formed a pit at the end of growth.

The images in Figure 4(c) and 4(d) provide a vision of the pit filling process through a large truncated pit using (a) $\vec{g}=11 \overline{2} 0$ and (b) $\vec{g}=0002$ near the [1 $\overline{1} 00]$ zone axis. There was also an image contrast at the $\{20 \overline{2} 1\}$ facet where the dislocations bent from the vertical direction to the [20 $\overline{2} 1]$ direction and then back to the [11 $\overline{2} 0]$ direction. Because TDs tend to intersect a free surface at $90^{\circ}$ in response to the image forces acting on the dislocation and to minimize the total dislocation line energy[29, 30], from the dislocation propagation track, growth evolution could be deduced. We could see that the nucleation islands met each other $200 \mathrm{~nm}$ above the substrate interface and grew laterally in the $\{20 \overline{2} 1\}$ and $\{10 \overline{1} \overline{2}\}$ surface, as well as vertically in the [11 0 ] direction. As growth proceeded, the growth front gradually changed from $\{20 \overline{2} 1\}$ to $(11 \overline{2} 0)$. Note that although there were many dislocations connected with the large pit, dislocation was not the cause of the pit but would be affected by its formation process, such as strong bending.

Figures 4(e) and 4(f) show the cross-sectional images of a large surface pit on c-plane GaN obtained in the same region but using (e) $\vec{g}=0002$ and (f) $\vec{g}=11 \overline{2} 0$ near the [1 $\overline{1} 00$ ] zone axis. The strain contrast caused by TD was more confined and the dislocation line was sharper compared with the a-plane GaN image because of the low defect density. The large surface pit was not associated with a single dislocation line but three pure screw dislocations, two mixed dislocations and two pure edge dislocations that propagated toward the $\{10 \overline{1} 1\}$ facet. There was a slight image contrast at two sides of the pit in Figure 4(f). Although the distortion between the two adjacent islands that formed the large pit and the dislocation bending of c-plane GaN were not as significant as that of the a-plane sample, their tendencies were similar. It could be concluded that the formation mechanism of the large surface pit was the same for both the a- and c-plane samples.

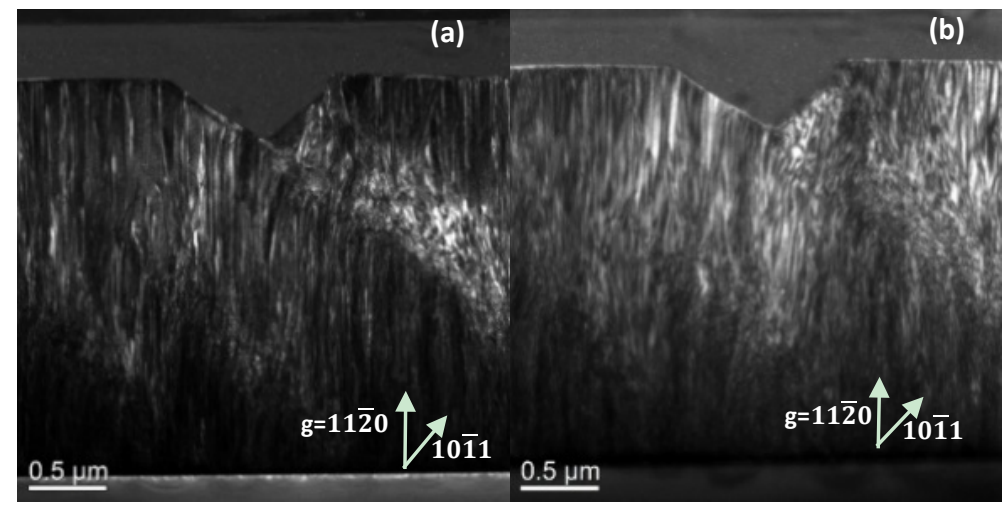



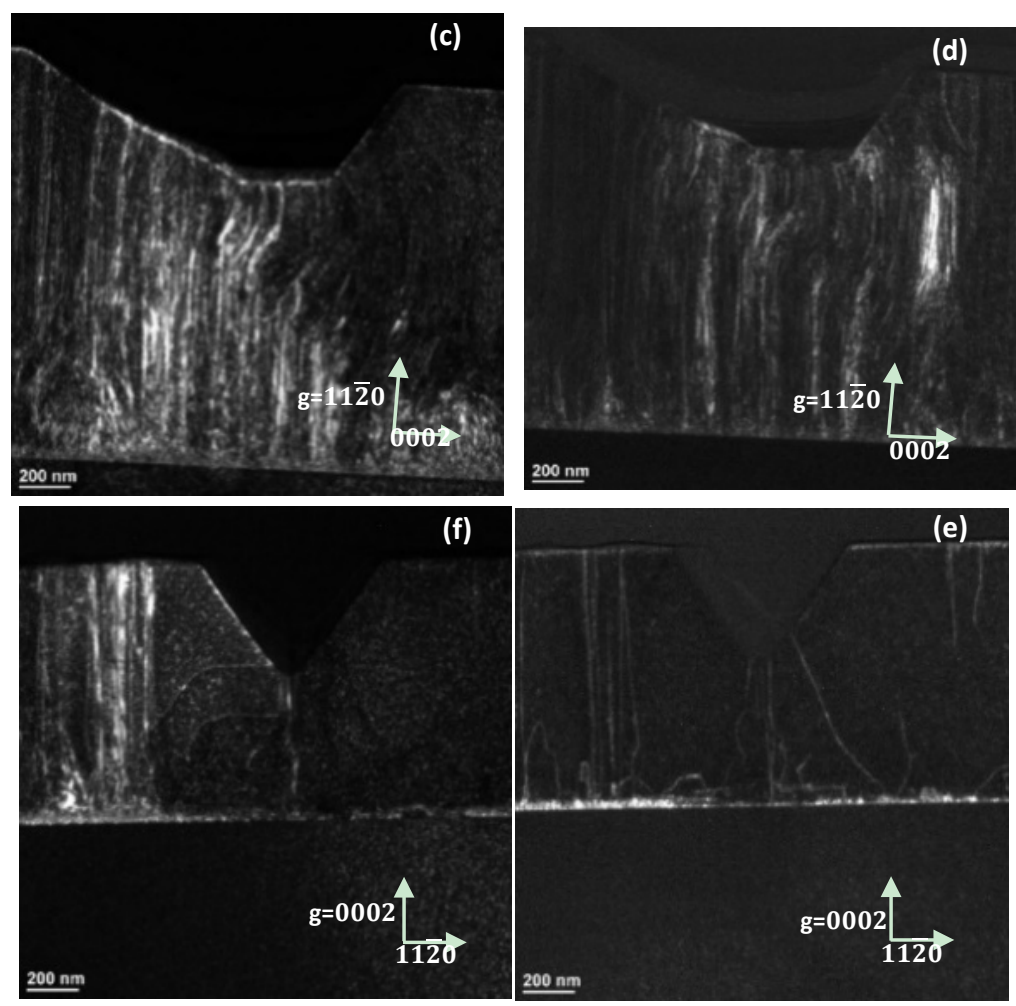

Fig.4. Cross-sectional weak beam dark-field TEM images of large surface pits for a-plane GaN (a) (d) and c-plane GaN (e) (f). Images were taken in the same region of one large pit in a-plane GaN using (a) $\vec{g}=11 \overline{2} 0$ and (b) $\vec{g}=10 \overline{1} 1$ near the $[01 \overline{1} 1]$ zone axis, and another pit using (c) $\vec{g}=11 \overline{2} 0$ and (d) $\vec{g}=0002$ near the

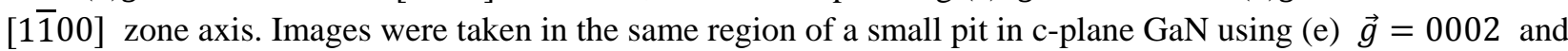
(f) $\vec{g}=11 \overline{2} 0$ near the $[1 \overline{1} 00]$ zone axis.

\section{Discussion}

The early work by Burton, Cabrera and Frank[26]predicted that surface pits may form when a TD with a screw component intersects the free surface of a crystal. The screw dislocation will cause a surface displacement equal to the component of the Burgers vector normal to the surface to achieve a balance between the surface energy and dislocation strain energy, which is manifested in a step line running outwards from the termination of the dislocation. The pit grows three-dimensionally from a continuous winding of surrounding steps in a helical pattern around the core to an ever-increasing relative depth. This prediction was demonstrated in c-plane GaN[15-18, 20, 22, 25]. A stepped morphology was the typical feature of c-plane GaN observed under AFM, and surface pits were usually found at the intersections of atomic surface steps shown as dark spots. The larger size of the pit, the more steps it was connected with ${ }^{[15]}$ and the greater surface roughness. For a-plane GaN, researchers have not correlated the surface pits with screw dislocations as far as we know, partially because no surface steps have found in an AFM image, and large surface pits and strips are the intensively reported morphologic feature. In addition, PDs and SFs are the majority of defects in a-plane GaN. However, based on our experimental results, surface pits with relative small size were connected with screw component dislocation at the apex. According to the pit radius equation Frank has deduced by balancing the elastic dislocation strain energy against the energy of the resulting free surfaces[26]:

$$
r_{0}=\frac{\mu|\vec{b}|^{2}}{8 \pi^{2} \gamma}
$$

where $\vec{b}$ is the component of the Burgers vector that parallel to the dislocation line, $\mu$ is the rigidity 
modulus, and $\gamma$ is surface free energy, a larger Burgers vector component along the dislocation line corresponds to a larger pit size. Since PD also has the component in the direction perpendicular to the surface, it should have the same surface termination mechanism as perfect screw dislocation, however, with smaller Burgers component parallel to the dislocation line, pit that produced by PD will be much smaller than that caused by perfect screw dislocation. This result demonstrated the prediction of Frank's theory in a-plane GaN. Spiral growth is the intrinsic behavior for screw dislocation and does not distinguish between epitaxial orientations, whether it is a-plane or c-plane. The pinned step lines may not have been observed because of the interference of strips, and the surface roughness was above the atomic level.

In addition to dislocation mediation, kinetic effect is also critical in determining the surface morphology of a heteroepitaxial film [5, 6, 8, 11, 12]. The kinetic process of island coalescence may produce surface pits for a-plane GaN, as well as c-plane GaN. Filling such surface pits to achieve a smooth coalesced film required faster lateral growth velocities including the $\{10 \overline{1} \overline{2}\},\{11 \overline{2} 2\}$, and $\{20 \overline{2} 1\}$ facets or the $\{10 \overline{1} 1\}$ and $\{000 \overline{1}\}$ facets that were in competition with the vertical growth of the $(11 \overline{2} 0)$ plane for a-plane GaN, and for c-plane GaNit required the lateral growth velocity of $\{10 \overline{1} 1\}$ competed with the vertical growth velocity of the (0001) plane, as observed in our experimental results. The anisotropic crystallographic difference between c-plane and a-plane GaN only determined whether it was a stepped or striped surface, a hexagonal or triangular pit, but did not determine the island coalescing degree. The large pit formation mechanism during island coalescing could be understood by the kinetic Wulff plot[10, 30, 31], which have applied the Wulff construction based on minimizing the total free energy principle under equilibrium conditions to the surface orientation-dependent growth velocity plot in the kinetically controlled growth regime. From the plot[10, 30], it could be observed that under low V/III, the ratio of the growth velocity between the inclined facets and $(11 \overline{2} 0)$ was larger, whereas the velocity ratio between the inclined facets and(0001)was smaller compared with the high V/III situation. Therefore, through reducing the V/III ratio, the large pit could be eliminated for a-plane GaN but appeared on the c-plane GaN surface. In addition, under high temperature, the growth velocity of $\{20 \overline{2} 1\}$ and $\{11 \overline{2} 2\}$ facets was approaching to that of $\{10 \overline{1} 1\}$ facets,so thatthe growth fronts in the lateral directions would change from $\{10 \overline{1} 1\}$ to $\{20 \overline{2} 1\}$ and $\{11 \overline{2} 2\}$. Thereafter, the triangular shaped pit transformed to a pentagonal pit.

The kinetic effect must also be considered in the formation mechanism of the small surface pits, because the size of the small pits varied together with the size of the large pits. Frank's model only considers a surface under equilibrium conditions. Actually, the pit size first theoretically calculated by Frank was in the sub-nanometer scale. Researchers believed that pit formation for c-plane GaN may be related to the segregation of local do pants, such as Si[16], Mg[17], In[19-21,24], around the dislocation core or even other impurities such as $\mathrm{O}$ [32]. When the concentration of the impurity atoms increases to a certain degree, it may slow down or inhibit the crystal growth on the inclined plane ${ }^{[19,27]}$. In our experiments, the samples were undoped; however, the formation of small pit son the dislocation termination was still found to be affected by the V/III ratio. A reduced V/III ratio may also enlarge the pit at the dislocation core by reducing the lateral vs. vertical growth rate ratio for c-plane $\mathrm{GaN}$, and for a-plane GaN, an increased V/III ratio may enlarge the pit. It should be noted that GaN epitaxial growth is a non-equilibrium kinetic process, but in sub-nanometer, it is still under equilibrium according to the local thermodynamic assumption in a dissipation system as proposed by P. Glansdorff[33], therefore, Frank's model is still workable in a small local range. We believe that the small pits resulting from screw dislocations would not completely eliminated from the surface, which was determined by its nature. However, the pit size might be in the atomic dimension such that it would be difficult to detect.

\section{Summary}

As the same material, the formation mechanisms of surface pits were the same for both a-plane and 
c-plane GaN. There were two factors governing thepit formation process: dislocation and the kinetic effect during growth. Screw dislocation resulted in pits with relatively small size on both the a- and c-plane GaN surfaces due to its intrinsic nature of spiral growth, whereas the kinetic process of the atomic reactions on the surface mainly controlled the size of the pit. Large surface pits were formed with a hexagonal shape on the c-plane surface and with a triangular or pentagonal shape on the a-plane surface, both as a result of incomplete island coalescence, governed by the kinetic process of the lateral growth velocities of the inclined facets that were in competition with the vertical growth velocity in the epitaxial orientation.

\section{Acknowledgement}

This work was supported by National Natural Science Foundation of China (Grant No. 11204009), and Beijing Municipal Natural Science Foundation (Grant No.4142005).

\section{Reference}

[1]S. Nakamura, The Roles of Structural Imperfections in InGaN-Based Blue Light-Emitting Diodes and Laser Diodes, Science 281 (1998) 956-961.

[2] S. Hu, S. Liu, Z. Zhang, H. Yan, Z. Gan, H. Fang, A novel MOCVD reactor for growth of high-quality GaN-related LED layers. J. Crystal Growth 415 (2015) 72-77.

[3] P. Waltereit, O. Brandt, A. Trampert, H.T. Grahn, J. Menniger, M. Ramsteiner, M. Reiche, K.H. Ploog, Nitride semiconductors free of electrostatic fields for efficient white light-emitting diodes, Nature 406 (2000) 865-868.

[4] S.R. Xu, J.C. Zhang, L.A. Yang, X. W. Zhou, Y. R. Cao, J.F. Zhang, J.S. Xue, Z.Y. Liu, J.C. Ma,

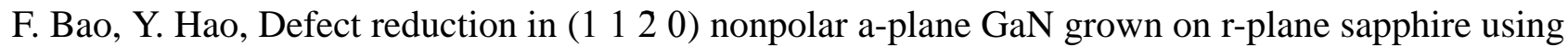
TiN interlayers, J. Crystal Growth 327 (2011) 94-97.

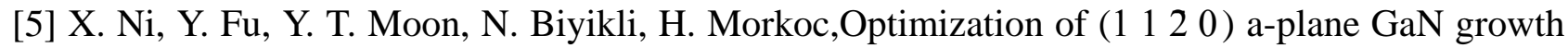
by MOCVD on (11-02) r-plane sapphire, J. Crystal Growth 290 (2006) 166-170.

[6] J. Zhang, W. Tian, F. Wu, Q.X. Wan, Z.J. Wang, J. Zhang, Y.L. Li, J.N. Dai, Y.Y. Fang, Z.H. Wu, C.Q. Chen, J.T. X, X.Y. Li,The effects of substrate nitridation on the growth of nonpolar a-plane $\mathrm{GaN}$ on r-plane sapphire by metalorganic chemical vapor deposition, Appl. Surface Science 307 (2014) 525-532.

[7] K.M. Song, J.M. Kim, B.K. Kang, C.S. Shin, C.G. Ko, B.H. Kong, H.K. Cho, D.H. Yoon, H. Kim, S.M. Hwang, Effect of growth pressure on the morphology evolution and doping characteristics in nonpolar a-plane GaN, Appl. Surface Science 258 (2012) 3565-3570.

[8] K.M. Song, J.M. Kim, D.H. Lee, C.S. Shin, C.G. Ko, B.H. Kong, H.K. Cho, D.H. Yoon, Growth behavior and growth rate dependency in LEDs performance for Mg-doped a-plane GaN, J. Crystal Growth 326 (2011) 135-139.

[9] T.J. Badcock, R. Hao, M.A. Moram, P. Dawson, M.J. Kappers, C.J. Humphreys, Properties of surface-pit related emission in a -plane InGaN/GaN quantum wells grown on $\mathrm{r}$-plane sapphire, Phys. Status. Solidi C 7 (2011) 2179-2181.

[10] Q. Sun, C.D. Yerino, T.S. Ko, Y.S. Cho, I.H. Lee, J. Han, M.E. Coltrin, Understanding nonpolar GaN growth through kinetic Wulff plots, J. Appl. Phys. 104 (2008) 093523.

[11] C.F. Johnston, M.J. Kappers, C.J. Humphreys, Microstructural evolution of nonpolar (11-20) GaN grown on (1-102) sapphire using a 3D-2D method, J. Appl. Phys. 105 (2009) 073102.

[12] J. L. Hollander, M.J. Kappers, C. McAleese, C.J. Humphreys, Improvements in aa-plane GaN crystal quality by a two-step growth process, Appl. Phys. Lett. 92 (2008) 101104. 
[13] D.X. Du, D.J. Srolovitz, Faceted dislocation surface pits, Acta Materialia 52 (2004) 3365-3374.

[14] J. Bai, T. Wang, P.J. Parbrook, I.M. Ross, A.G. Cullis, V-shaped pits formed at the GaN/AlN interface, J. Crystal Growth 289 (2006) 63-67.

[15] P. Q. Miraglia, E.A. Preble, A.M. Roskowski, S. Einfeldt, R.F. Davis, Helical-type surface defects in GaN thin films epitaxially grown on GaN templates at reduced temperatures, J. Crystal Growth 253 (2003) 16-25.

[16] K.S. Son, D.G. Kim, H.K. Cho, K. Lee, S. Kim, K. Park, Formation of V-shaped pits in GaN thin films grown on high temperature GaN, J. Crystal Growth 261 (2004) 50-54.

[17] Z. Liliental-Weber, Y. Chen, S. Ruvimov, J. Washburn, Formation Mechanism of Nanotubes in GaN, Phys. Rev. Lett. 79 (1997) 2835-2838.

[18] B. Heying, E.J. Tarsa, C.R. Elsass, P. Fini, S.P. DenBaars, J.S. Speck,Dislocation mediated surface morphology of GaN, J. Appl. Phys. 85 (1999) 6470-6476.

[19] Y. Chen, T. Takeeuchi, H. Amano, I. Akasaki, N. Yamada, Y. Kaneko, S.Y. Wang, Pit formation in GaInN quantum wells, Appl. Phys. Lett. 72 (1998) 710-712.

[20] C.B. Soh, S.J. Chua, S. Tripathy, W. Liu, D.Z. Chi, The influence of V defects on luminescence properties of AlInGaN quaternary alloys , J. Phys.:Condens. Matter 17 (2005) 729-736.

[21] J. E. Northrup, L. T. Romano, J. Neugebauer, Surface energetics, pit formation, and chemical ordering in InGaN alloys, Appl. Phys. Lett. 74 (1999) 2319-2321.

[22] Y.B. Tao, Z.Z. Chen, T.J. Yu, Y. Yin, X.N. Kang, Z.J. Yang, G.Z. Ran, G.Y. Zhang, Improvement of structural and luminescence properties in InGaN/GaN multiple quantum wells by symmetrical thin low temperature-GaN layers, J. Crystal Growth 318 (2011) 509-512.

[23] D.S. Jiang, D.G. Zhao, H. Yang, Optical analysis of dislocation-related physical processes in GaN-based epilayers, Phys. Stat. Sol. B 244 (2007) 2878-2891.

[24] J.P. Liu, Y.T. Wang, H. Yang, D.S. Jiang, U. Jahn, K.H. Ploog, Investigations on V-defects in quaternary AlInGaN epilayers, Appl. Phys. Lett. 84 (2004) 5449-5451.

[25] B. Moran, F. Wu, A.E. Romanov, U.K. Mishra, S.P. Denbaars, J.S. Speck, Pyramids and domes in the InAs/GaAs(0 0 1) and Ge/Si(0 0 1) systems,J. Cryst. Growth 273 (2004) 38-45.

[26] F. C. Frank, Capillary equilibria of dislocated crystals, Acta Crystallogr. 4 (1951) 497-501.

[27] X.H. Wu, C.R. Elsass, A. Abare, M. Mack, S. Keller, P.M. Petroff, S.P. DenBaars, J.S. Speck, Structural origin of V-defects and correlation with localized excitonic centers in InGaN/GaN multiple quantum wells, Appl. Phys. Lett. 72 (1998) 692-694.

[28] B.H. Kong, Q. Sun, J. Han, I. Lee, H.K. Cho, Classification of stacking faults and dislocations observed in nonpolar a-plane GaN epilayers using transmission electron microscopy, Appl. Surface Science 258 (2012) 2522-2528.

[29] R. Hao, M. J. Kappers, M. A. Moram, C. J. Humphreys, Defect reduction processes in heteroepitaxial non-polar a-plane GaN films, J. Cryst. Growth 337 (2011) 81-86.

[30] Q. Sun, C.D. Yerino, B. Leung, J. Han, M.E. Coltrin, Understanding and controlling heteroepitaxy with the kinetic Wulff plot: A case study with GaN, J. Appl. Phys. 110 (2011) 053517.

[31] D. Du, D.J. Srolovitz, M.E. Coltrin, C.C. Mitchell, Systematic Prediction of Kinetically Limited Crystal Growth Morphologies, Phys. Rew. Lett. 95 (2005) 155503.

[32] R.Y. Korotkov, F. Niu, J.M.Gregie, B.W. Wessels, Investigation of the defect structure of GaN heavily doped with oxygen, Physica B 308 (2001) 26-29. 
[33] Glansdorff P and Prigogine I 1971 Thermodynamic theory of structure, stability and fluctuations (London: John Wiley \& Sons Ltd) pp. 14-16. 